\title{
HOMOTOPY GROUPS OF COMPACT ABELIAN GROUPS
}

\author{
EDGAR ENOCHS
}

The object of this paper is to prove the following:

Theorem. For any compact Abelian group $G, \pi_{n}(G)=0$ for $n \geqq 2$ and $\pi_{1}(G)$ is isomorphic to the group of homomorphisms of $G^{*}$ (the Pontrjagin dual of $G$ ) into $Z$ (the group of integers).

Preliminaries. $B_{n}$ (resp. $S_{n-1}$ ) will denote the subset of $R^{n}$ consisting of those $x$ such that $\|x\| \leqq 1$ (resp. $\|x\|=1$ ). $x_{0}$ will denote the point $(1,0,0, \cdots, 0)$ of $R^{n}$ and $T$ will denote $S_{1}$ made into a topological group by using complex multiplication. All groups will be assumed to be Abelian.

For a based topological space $X$ and a topological group $G$, let $\mathfrak{e}(X, G)$ denote the set of maps (i.e., base point preserving continuous maps) of $X$ into $G$ (where $G$ is considered to be a based topological space with base point 0$)$. In an obvious fashion, $\mathfrak{C}(X, G)$ can be endowed with a group structure. In case $X$ is also a topological group (considered as a based topological space with base point 0 ) let $\operatorname{Hom}(X, G)$ denote the subgroup of $\mathfrak{e}(X, G)$ consisting of those maps which are homomorphisms. The spaces $B_{n}$ and $S_{n-1}$ will all be assumed to be based with $x_{0}$ as a base point.

If $G$ is a discrete or compact group we let $G^{*}$ denote the group $\operatorname{Hom}(G, T)$ endowed with the topology of compact convergence. If $G$ is compact (resp. discrete) then it is known that $G^{*}$ is discrete (resp. compact). Also for two discrete or two compact groups $G_{1}$ and $G_{2}$ we have an isomorphism from the group $\operatorname{Hom}\left(G_{1}, G_{2}\right)$ onto $\operatorname{Hom}\left(G_{2}{ }^{*}, G_{1}{ }^{*}\right)$ where an element $f \in \operatorname{Hom}\left(G_{1}, G_{2}\right)$ is mapped onto its transpose $f^{*}$. Furthermore a sequence of compact (or discrete) groups

$$
G_{1} \stackrel{f}{\rightarrow} G_{2} \stackrel{g}{\rightarrow} G_{3}
$$

is exact if and only if the sequence

$$
G_{3}^{*} \stackrel{g^{*}}{\rightarrow} G_{2}^{*} \stackrel{f^{*}}{\rightarrow} G_{1}^{*}
$$

is exact (see Weyl [1]).

Using a general existence proof (see Bourbaki [2, p. 44, Theorem CST 22]), it can be shown that for every based topological space $X$

Presented to the Society, November 16, 1963; received by the editors February 27, 1963 and, in revised form, July 3, 1963. 
there is a compact group $\tilde{X}$ and a map $i: X \rightarrow \tilde{X}$ such that if $\phi: X \rightarrow G$ is any map where $G$ is a compact group, then there is a unique map $f: \tilde{X} \rightarrow G$ such that $f \circ i=\phi$. Thus for any compact group $G$ we get a natural isomorphism between the groups $\operatorname{Hom}(\tilde{X}, G)$ and $\mathcal{e}(X, G)$. We say that $i$ is a free compact group on $X$. It is easy to see that $i$ is an injection if $X$ is completely regular since, if $G$ is taken to be the product of sufficiently many copies of $T$, then $\phi$ can be chosen to be an injection.

Now let $j_{n}: S_{n} \rightarrow \widetilde{S}_{n}$ and $k_{n}: B_{n} \rightarrow \widetilde{B}_{n}$ be free compact groups on $S_{n}$ and $B_{n}$, respectively, and let $h_{n}$ be the unique continuous homomorphism from $\widetilde{S}_{n}$ into $\widetilde{B}_{n}$ such that

$$
\begin{array}{cr}
S_{n} \stackrel{i_{n}}{\longrightarrow} B_{n+1} \\
\quad \downarrow j_{n} \quad \downarrow k_{n} \\
\tilde{S}_{n} \stackrel{h_{n}}{\longrightarrow} \widetilde{B}_{n+1}
\end{array}
$$

is commutative where $i_{n}$ is the canonical injection.

Now for any topological group $G, \pi_{n}(G)$ is isomorphic to the quotient of the group $\mathrm{e}\left(S_{n}, G\right)$ by the subgroup of $\mathfrak{e}\left(S_{n}, G\right)$ consisting of those elements that can be continuously extended to $B_{n+1}$ (see $\mathrm{Hu}[3$, p. 139, Example $G]$ ). But this subgroup is precisely the image of $\mathfrak{C}\left(B_{n+1}, G\right)$ in $\mathcal{e}\left(S_{n}, G\right)$ under the restriction map. Thus $\pi_{n}(G)$ equals the cokernel of the restriction map

$$
\mathfrak{C}\left(B_{n+1}, G\right) \rightarrow \mathfrak{C}\left(S_{n}, G\right) .
$$

But we have a commutative diagram

$$
\begin{gathered}
\mathcal{e}\left(B_{n+1}, G\right) \longrightarrow \\
\uparrow \\
\operatorname{Hom}\left(\tilde{B}_{n+1}, G\right) \stackrel{\operatorname{Hom}\left(h_{n}, G\right)}{\longrightarrow} \operatorname{Hom}\left(\tilde{S}_{n}, G\right)
\end{gathered}
$$

where the vertical maps are the natural isomorphisms and the top map is the restriction map. Thus for any compact group $G, \pi_{n}(G)$ and $\operatorname{Coker}\left(\operatorname{Hom}\left(h_{n}, G\right)\right)$ are isomorphic. But

$$
\begin{gathered}
\operatorname{Hom}\left(\widetilde{B}_{n+1}, G\right) \stackrel{\operatorname{Hom}\left(h_{n}, G\right)}{\longrightarrow} \operatorname{Hom}\left(S_{n}, G\right) \\
\downarrow \\
\operatorname{Hom}\left(G^{*}, \widetilde{B}_{n+1}^{*}\right) \stackrel{\operatorname{Hom}\left(G^{*}, h_{n}^{*}\right)}{\longrightarrow} \operatorname{Hom}\left(G^{*}, \widetilde{S}_{n}{ }^{*}\right)
\end{gathered}
$$

is commutative where the vertical mappings are the transpose mappings (hence are isomorphisms). Thus $\pi_{n}(G)$ is isomorphic to 
$\operatorname{Coker}\left(\operatorname{Hom}\left(G^{*}, h_{n}^{*}\right)\right)$. We will try to show that $\operatorname{Coker}\left(\operatorname{Hom}\left(G^{*}, h_{n}{ }^{*}\right)\right)$ is isomorphic to $\operatorname{Hom}\left(G^{*}\right.$, Coker $\left.h_{n}^{*}\right)$

Proof of TheOREM. Let

$$
0 \rightarrow M_{n} \stackrel{t_{n}}{\rightarrow} \tilde{S}_{n} \stackrel{h_{n}}{\rightarrow} \tilde{B}_{n+1} \stackrel{p_{n}}{\rightarrow} N_{n} \rightarrow 0
$$

be an exact sequence of compact groups. Then

$$
0 \longleftarrow M_{n}^{*} \stackrel{t_{n}^{*}}{\longleftarrow} \widetilde{S}_{n}^{*} \stackrel{h_{n}^{*}}{\longleftarrow} \tilde{B}_{n+1}^{*} \stackrel{p_{n}^{*}}{\longleftarrow} N_{n}^{*} \longleftarrow 0
$$

is an exact sequence of discrete groups. Thus $M_{n}^{*}$ is isomorphic to Coker $h_{n}^{*}$. But

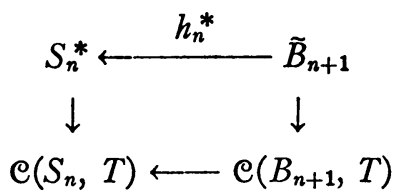

is commutative with the vertical maps, the natural isomorphisms and the bottom map, the restriction map. But then Coker $h_{n}{ }^{*}$ is isomorphic to $\pi_{n}(T)$ since the cokernel of the bottom map is $\pi_{n}(T)$. Thus $M_{1}^{*} \cong Z$ and $M_{n}^{*}=0$ for $n \geqq 2$ since $\pi_{1}(T)=Z$ and $\pi_{n}(T)=0$ for $n \geqq 2$.

Furthermore we claim $N_{n}^{*}$ is divisible for $n \geqq 1$. For $N_{n}^{*}$ is isomorphic to the kernel of the map

$$
\tilde{B}_{n+1}^{*} \stackrel{h_{n}^{*}}{\longrightarrow} \tilde{S}_{n}^{*},
$$

hence to the kernel $H$ of the restriction map

$$
\mathfrak{C}\left(B_{n-1}, T\right) \rightarrow \mathfrak{e}\left(S_{n}, T\right) .
$$

Let $B_{n+1} / S_{n}$ be the quotient space of $B_{n+1}$ by the equivalence relation which identifies all the points in $S_{n}$. Then $H$ is isomorphic to $\mathfrak{e}\left(B_{n+1} / S_{n}, T\right)$. But $B_{n+1} / S_{n}$ is homeomorphic to $S_{n+1}$. So $H$ is isomorphic to $\mathfrak{C}\left(S_{n+1}, T\right)$. But $\pi_{n+1}(T)=0$ for $n \geqq 1$, hence any map $S_{n+1} \rightarrow T$ is homotopic to a constant mapping. Thus, the map $S_{n+1} \rightarrow T$ can be "factored through $R$ " by the exponential map $R \rightarrow T$ (see Dieudonné $\left[4\right.$, p. 248]). Thus $\mathrm{e}\left(S_{n+1}, T\right)$ is isomorphic to a quotient of $\mathfrak{e}\left(S_{n+1}, R\right)$ which is clearly divisible. Thus $\mathcal{e}\left(S_{n+1}, T\right)$ is divisible and so $H$ and $N_{n}^{*}$ are divisible.

Recalling that $M_{n}^{*}=Z$ or 0 we see that the sequence

$$
0 \longleftarrow-M_{n}^{*} \stackrel{t_{n}^{*}}{\longleftarrow} S_{n}^{*} \stackrel{h_{n}^{*}}{\longleftarrow} \dot{B}_{n+1}^{*} \stackrel{p_{n}^{*}}{\longleftarrow} N_{n}^{*} \leftarrow-0
$$


is split exact, i.e., it is exact and the kernel of each map is a direct summand of the domain of the map. Hence, for a compact group $G$,

$$
\begin{gathered}
0 \longleftarrow \operatorname{Hom}\left(G^{*}, M_{n}^{*}\right) \stackrel{\operatorname{Hom}\left(G^{*}, t_{n}^{*}\right)}{\longleftarrow} \operatorname{Hom}\left(G^{*}, \tilde{S}_{n}^{*}\right) \stackrel{\operatorname{Hom}\left(G^{*}, h_{n}^{*}\right)}{\longleftarrow} \\
\longleftarrow \operatorname{Hom}\left(G^{*}, \tilde{B}_{n+1}^{*}\right) \stackrel{\operatorname{Hom}\left(G^{*}, p_{n}^{*}\right)}{\longleftarrow} \operatorname{Hom}\left(G^{*}, N_{n}^{*}\right) \longleftarrow-0
\end{gathered}
$$

is exact (even split exact). Thus $\operatorname{Coker}\left(\operatorname{Hom}\left(G^{*}, h_{n}^{*}\right)\right)$ is isomorphic to $\operatorname{Hom}\left(G^{*}, M_{n}^{*}\right)$. But $\operatorname{Coker}\left(\operatorname{Hom}\left(G^{*}, h_{n}^{*}\right)\right)$ is isomorphic to $\pi_{n}(G)$. Hence $\pi_{n}(G)=0$ for $n \geqq 2$ since $M_{n}^{*}=0$ for $n \geqq 2$ and $\pi_{1}(G)$ is isomorphic to $\operatorname{Hom}\left(G^{*}, Z\right)$ since $M_{1}^{*} \cong Z$. This completes the proof.

\section{BIBLIOGRAPHY}

1. A. Weyl, L'intégration dans les groupes topologiques et ses applications, Hermann, Paris, 1951.

2. N. Bourbaki, Theorie des ensembles, Hermann, Paris, 1957; Chapter 4.

3. S. Hu, Homotopy theory, Academic Press, New York, 1959.

4. J. Dieudonné, Foundations of modern analysis, Academic Press, New York, 1960.

University of South Carolina 